\title{
Brain correlates of memory dysfunction in alcoholic Korsakoff's syndrome
}

P J Visser, L Krabbendam, F R J Verhey, P A M Hofman, W M A Verhoeven, S Tuinier, A Wester, Y W M M Van Den Berg, L F M Goessens, Y D Van Der Werf, J Jolles

Institute of Brain and Behavior, Department of Psychiatry, University of

Maastricht, Maastricht, The Netherlands P J Visser L Krabbendam F R J Verhey J Jolles

Department of Radiology, Academisch Ziekenhuis Maastricht, Maastricht, The

Netherlands

P A M Hofman

Vincent van Gogh

Institute, Venray, The

Netherlands

W M A Verhoeven

$S$ Tuinier

A Wester

Y W M M Van Den Berg

L F M Goessens

Graduate School for

Neurosciences

Amsterdam,

Department of

Anatomy, Vrije

Universiteit,

Amsterdam, The

Netherlands

Y D Van Der Werf

Correspondence to: Dr P J Visser, Academische Afdeling Neuropsychologie, PMS Vijverdal, PO Box 88, 6200 AB Maastricht, The

Netherlands.

Received 18 March 1999 and in revised form

12 July 1999

Accepted 22 July 1999

\begin{abstract}
Objectives-To investigate the relation between anterograde amnesia and atrophy of brain structures involved in memory processing in alcoholic Korsakoff's syndrome.

Methods-The volume of brain structures involved in memory processing was measured with MRI from 13 subjects with Korsakoff's syndrome, 13 subjects with chronic alcoholism without Korsakoff's syndrome, and 13 control subjects. The brain structures analysed were the hippocampus, the parahippocampal gyrus, the mamillary bodies, the third ventricle, and the thalamus. Brain volumes were correlated with the delayed recall of a verbal learning test.

Results-Compared with subjects with chronic alcoholism and control subjects, subjects with Korsakoff's syndrome had a reduced volume of the hippocampus, the mamillary bodies, and the thalamus, and enlargement of the third ventricle. The impairment of delayed recall correlated with the volume of the third ventricle $(r=-0.55, p=0.05)$ in the Korsakoff group. Conclusions-Anterograde amnesia in alcoholic Korsakoff's syndrome is associated with atrophy of the nuclei in the midline of the thalamus, but not with atrophy of the mamillary bodies, the hippocampus, or the parahippocampal gyrus.
\end{abstract}

(F Neurol Neurosurg Psychiatry 1999;67:774-778)

Keywords: Korsakoff's syndrome; alcoholism; memory; magnetic resonance imaging

Anterograde amnesia is one of the most prominent features of Korsakoff's syndrome. ${ }^{1}$ The brain correlate of the anterograde amnesia in Korsakoff's syndrome is still controversial. Neuroimaging and neuropathological studies have indicated that several structures may be the brain substrate for anterograde amnesia including the mediodorsal nucleus of the thalamus, ${ }^{23}$ the parataenial nucleus of the thalamus, ${ }^{45}$ the mamillary bodies,${ }^{67}$ the frontal cortex ${ }^{3}$ the cingulate gyrus, ${ }^{8}$ the nucleus basalis of Meynert, ${ }^{9}$ the nucleus coeruleus, ${ }^{5}$ the hippocampus, ${ }^{10} 11$ and the amygdala. ${ }^{10}{ }^{11}$ It is surprising that none of the neuroimaging studies so far made use of volumetry on MRI. The advantage of volumetry is that it can demonstrate correlations between the volume of brain structures and memory impairment. ${ }^{12}$ Another advantage of this technique is that the volume of brain structures and cognitive function can be measured more or less at the same time, by contrast with postmortem studies.

The aim of the present study was to investigate in subjects with alcoholic Korsakoff's syndrome the relation between anterograde amnesia and the volume of brain structures that are known to be involved in memory processing, focusing on structures of the limbic system. We measured the volume of the hippocampus, the parahippocampal gyrus, the mamillary bodies, and the thalamus on high resolution MRI. We also measured the volume of the third ventricle because dilatation of this structure may reflect atrophy of nuclei in the midline of the thalamus, including the mediodorsal nucleus of the thalamus. ${ }^{13}$ All the subjects with Korsakoff's syndrome were alcoholics. Because prolonged alcohol intake is neurotoxic, we included a control group of alcoholics without Korsakoff's syndrome to identify brain abnormalities that are due to thiamine deficiency and not to prolonged alcohol intake. A second control group consisted of matched healthy volunteers. Care was taken to match the three groups on an individual basis for age, sex, and education. We first determined which brain structures were significantly different between alcoholics with Korsakoff's syndrome and alcoholics without the syndrome. We then correlated the volume of the structures that were significantly different in subjects with Korsakoff's syndrome with the severity of anterograde amnesia in these patients.

\section{Methods}

SUBJECTS

Thirteen patients with alcohol induced persisting amnestic disorder (Korsakoff's syndrome; DSM-IV 291.1 14 ), 13 patients with alcohol dependence (DSM-IV 303.90 ${ }^{14}$ ), and 13 healthy control subjects participated in the study. These groups are referred to as the Korsakoff group, alcoholic group, and control group, respectively. Patients were diagnosed by a multidisciplinary team consisting of a neuropsychiatrist, a neuropsychologist, and a neurologist. The patients were recruited from specialised departments for patients with Korsakoff's syndrome or chronic alcoholism from the Vincent van Gogh Institute for Mental Health in Venray, the Netherlands. All patients were abstinent for at least 1 month. The control subjects were recruited via newspaper advertisements. Exclusion criteria for all subjects were: age older than 56 years (because of the interaction between age and alcohol related brain damage ${ }^{15}$ ), an intelligence 
Table 1 Subject characteristics

\begin{tabular}{llll}
\hline & Korsakoff & Alcoholic & Control \\
\hline No & 13 & 13 & 13 \\
Age (y) & $45.7(6.3)$ & $45.9(6.1)$ & $45.9(5.6)$ \\
Sex ratio & $11 \mathrm{M}: 2 \mathrm{~F}$ & $11 \mathrm{M}: 2 \mathrm{~F}$ & $11 \mathrm{M}: 2 \mathrm{~F}$ \\
Education (y) & $10.8(3.2)$ & $10.9(3.1)$ & $11.4(2.7)$ \\
Alcohol misuse (y) & $17.1(7.5)$ & $17.7(8.0)$ & 0 \\
IQ score & $104(14.9)$ & $112(10.6)$ & $111(7.4)$ \\
Delayed recall (z score) & $-2.82(0.61)^{\star}$ & $-0.09(1.2)$ & $-0.01(1.1)$ \\
\hline
\end{tabular}

Values are means (SD)

${ }^{\star} \mathrm{p}<0.001$ compared with alcoholic group and control group.

quotient below 80 (to exclude subjects with generalised cognitive impairment), the use of psychotropic medication, the presence of depression, psychotic disorders, anxiety disorders, dementia, diabetes mellitus, liver disease, other CNS diseases, and cardiac, pulmonary, or endocrine diseases that could affect cognitive functioning. Exclusion criteria for control subjects also included a history of alcohol dependence or a current intake or a history (longer than 1 month) of alcohol intake of 28 or more units a week. Subjects with Korsakoff's syndrome who were not amnestic on neuropsychological testing were also excluded. Written informed consent was obtained from all subjects.

The three groups were matched for age, sex, and education. Subject characteristics are shown in table 1 . The duration of alcohol misuse was estimated from the history of the patient and an informed other person, and by examining medical charts. No differences existed in the duration of alcohol misuse between the subjects with Korsakoff's syndrome and the subjects with chronic alcoholism (table 1).

NEUROPSYCHOLOGICAL METHODOLOGY

The neuropsychological assessment has been described in detail elsewere. ${ }^{16}$ Anterograde amnesia was assessed with the delayed recall of the auditory verbal learning test. ${ }^{17}$ Fifteen words were presented five times and after each presentation the subject was asked to reproduce as many words as possible. After $20 \mathrm{~min}$ utes, during which non-verbal tests were performed, the delayed recall was tested. The data are expressed as $\mathrm{z}$ scores. The $\mathrm{z}$ score is the number of SDs that the score deviates from the expected score in a normal population of a given age, sex, and education. The $\mathrm{z}$ scores were based on a reference population of 1870 normal and healthy subjects randomly selected from a registry of general practitioners. ${ }^{18}{ }^{19}$ As expected, the Korsakoff group performed significantly worse than the alcoholic group and the control group on the delayed recall task (table 1). No differences were found between the alcoholic group and the control group.

The shortened form of the Wechsler adult intelligence scale $^{20}$ was administered to the patient groups to obtain a measure of general intelligence, and the shortened form of an equivalent Dutch intelligence test, the Groningen intelligence test, ${ }^{21}$ was administered to the control group for the same purpose. No significant differences existed between the three groups (table 1).
MRI METHODOLOGY

A 3D volumetric scan ( $T 1$ weighted, fast field echo, TR $24 \mathrm{~ms}$, TE $7 \mathrm{~ms}$, flip angle $30^{\circ}$, number of averages $=2$, FOV $230 \mathrm{~mm}$, resolution $256 \times 154$ ), and an inversion recovery (IR) scan (TR $2107 \mathrm{~ms}$, TE $18 \mathrm{~ms}$, turbofactor=3, flip angle $90^{\circ}$, number of averages $=2$, FOV 230 $\mathrm{mm}$, resolution $256 \times 177$ ) were made on a 1.5 Tesla scanner (Gyroscan ACS-II, Philips). The slice thickness of the $3 \mathrm{D}$ volumetric scan was $1.5 \mathrm{~mm}$ and the scan axis was coronal, perpendicular to the intercommissural line. The slice thickness of the IR scan was $3 \mathrm{~mm}$ and the scan axis was coronal, perpendicular to the long axis of the hippocampus. The thalamus was measured on the IR scan and all other structures on the $3 \mathrm{D}$ volume scan.

Data were transferred to a SUN workstation and the regions of interest were measured with ShowImage (developed at the Department of Clinical Physics and Informatics, VrijeUniversiteit, Amsterdam, The Netherlands). A seed function was used to measure the third ventricle. The cut off level between CSF and brain was visually adjusted by the rater on each slice. The other structures were manually traced with a mouse driven cursor. All measurements were taken in a rostrocaudal direction. The volumes of the left side and right side were added in all analyses as the pathological changes in Korsakoff syndrome and alcoholism are bilateral. ${ }^{22}$ The volume of the brain structures was calculated by multiplying the surface area of each region of interest by the slice thickness and summing the volumes of all slices on which the structure was measured. Measurements were done with reference to several anatomical atlases. ${ }^{23-25}$ Each structure was measured by one rater who was blinded to all clinical information.

\section{METHODOLOGY OF BRAIN MEASUREMENTS \\ Hippocampus}

Measurements started with the slice on which both the semianular sulcus and a notch between the amygdala and the hippocampus in the medial wall of the lateral ventricle were visible. $^{26}$ Then every second slice was measured. The last slice was the slice before the slice on which the crura of the fornices were visible. On average 10 (range $8-13$ ) slices on each side were measured. The measurements included the hippocampus proper, the dentate gyrus, the alveus, and the portion of the subiculum which lies directly underneath the hippocampus. ${ }^{26}$

\section{Parahippocampal gyrus}

The same slices on which the hippocampus was measured were used, except for the last slice in order not to include the isthmus of the cingulate gyrus. The upper boundary was the hippocampus or the transverse fissure and the lateral boundary was the collateral sulcus. If this sulcus was not visible, a straight line was drawn perpendicular to the temporal stem through the centre of the first gyrus at the medial site of the temporal stem. The brain tissue medial to this line was considered as the parahippocampal gyrus. The lower and medial 
boundaries consisted of CSF or the tentorium cerebelli at the posterior slices.

\section{Mamillary bodies}

The mamillary bodies were measured on four or five consecutive slices. On the first slice the mamillary bodies appeared as a bulge in the floor of the third ventricle. On the next two slices the mamillary bodies had an ovoid shape and on the last slices they were small thickenings in the floor of the third ventricle or the medial wall of the hypothalamus. The left and right mamillary body were in most cases measured together.

\section{Third ventricle}

Measurements started with the slice on which the optic chiasma was connected to the hypothalamus. The last slice was the slice on which the posterior commissure was visible. On average 17 (range 15-20) slices were measured. The upper boundary on the slices on which the fornix was visible consisted of a straight line from the most superior point of the left and right side of the thalamus to the fornix. When the fornix was not present, a horizontal line was drawn between the upper medial border of the left and the right part of the thalamus where the distance between the two parts was shortest. The lateral and inferior boundaries consisted of the the thalamus or hypothalamus.

\section{Thalamus}

Measurements started one slice after the anterior commissure and stopped when the thalamus could no longer be detected. On average 10 (range 8-12) slices on each side were measured. The lateral boundary consisted of the internal capsule, the upper boundary of the lateral ventricles, and the medial boundary of the third ventricle. The columns of the fornix, the mamillary bodies, and the nucleus ruber were excluded. The medial and lateral geniculate bodies were included only when there was no white matter tract between these structures and the thalamus.

\section{Intracranial area}

The cranial area was measured on three slices: on the first slice on which the third ventricle was measured, on the second slice on which the mamillary bodies were measured, and on the last slice on which the third ventricle was measured. The volume of each section was derived by tracing the outline of the supratentorial compartment, following the dural and tentorial margins.

\section{Intraobserver variability}

The structures on 10 scans were remeasured. Pearson's correlation coefficient between the first and second measurements was 0.91 for the hippocampus, 0.91 for the parahippocampal gyrus, 0.98 for the third ventricle, 0.90 for the whole thalamus, 0.86 for the mamillary bodies, and 0.99 for the intracranial area. These correlations indicate a high level of intrarater agreement for all measurements.
Correction for age, sex, intracranial area, number of slices, and years of alcohol misuse

The years of alcohol misuse did not correlate with any of the brain volumes in the Korsakoff or alcoholic group and therefore we did not correct for it. The number of slices correlated with the total volume of the hippocampus, the parahippocampal gyrus, the third ventricle, and the thalamus. The number of slices on which the hippocampus and parahippocampal gyrus were measured did not differ between the Korsakoff group, alcoholic group, or control group. To reduce the variance of the data we corrected for the number of slices. This correction reduced the SD in the total group by $21 \%$ (parahippocampal gyrus) and 33\% (hippocampus), but the average volume in both the total group and the subgroups remained the same as that without correction for the number of slices. Because the thalamus and the third ventricle could be measured on significantly fewer slices in the Korsakoff group $(p=0.04)$ than in the control group, which possibly resulted from atrophy of the thalamus, we did not correct for the number of slices for these structures.

The correction for age, sex, intracranial area, and number of slices was based on a population of 60 healthy people ranging in age from 21 to 82 years (average age 56 (SD 15.9) years). These subjects were recruited by newspaper advertisements and included the subjects who were control subjects in the present study. Regression was performed with the brain structure as dependent variable and intracranial area, age, number of slices (only with hippocampus and parahippocampal gyrus), sex, the interaction term age by sex (because of reported differences in aging between men and women ${ }^{27}$ ), and age square (only with the third ventricle) as independent variables. Variables and interaction terms that were significant at the $p=0.05$ level were included in the final regression model. An expected volume for each individual was calculated on the basis of the constant and the $\beta$ coefficients from the final regression equation. The expected volume was subtracted from the observed volume. The difference (residue) was used for all analyses. The data are presented in table 2 as follows. We calculated an expected brain volume for a subject with the average study age (46 years), male sex, average number of slices on which the hippocampus and parahippocampus were measured, and average intracranial area. To this expected volume we added the residue of the study subjects.

STATISTICAL ANALYSIS

The data were analysed using SPSS for the Macintosh 4.0 (SPSS Inc, Chigaco, IL, USA). Categorical data were analysed by $\chi^{2}$ test. Group comparisons of continuous data were analysed with a $t$ test. Linear regression analysis was used to analyse the relation between memory score and brain volume. All tests were two tailed, and the significance level was set at 0.05 . 
Table 2 Brain volumes

\begin{tabular}{|c|c|c|c|c|c|c|}
\hline & \multirow[b]{2}{*}{ Korsakoff (Kors) } & \multirow[b]{2}{*}{ Alcoholic (Alc) } & \multirow[b]{2}{*}{ Control (Con) } & \multicolumn{3}{|l|}{$p$ Value } \\
\hline & & & & Kors $v$ Alc & Kors v Con & Alc v Con \\
\hline Hippocampus & $4.27(0.28)$ & $4.55(0.35)$ & $4.52(0.23)$ & 0.03 & 0.02 & 0.81 \\
\hline Parahippocampal gyrus & $6.15(0.44)$ & $6.15(0.45)$ & $6.46(0.40)$ & 1.0 & 0.07 & 0.07 \\
\hline Mamillary bodies & $0.049(0.011)$ & $0.068(0.015)$ & $0.069(0.012)$ & 0.001 & $<0.001$ & 0.89 \\
\hline Third ventricle & $1.79(0.33)$ & $1.30(0.44)$ & $1.04(0.28)$ & 0.004 & $<0.001$ & 0.08 \\
\hline Thalamus & $12.0(1.2)$ & $13.5(1.1)$ & $13.3(1.4)$ & 0.003 & 0.02 & 0.69 \\
\hline Intracranial area & $33.0(2.5)$ & $33.1(2.1)$ & $33.9(1.5)$ & 0.91 & 0.24 & 0.25 \\
\hline
\end{tabular}

Values are means (SD) in $\mathrm{cm}^{3}$.

Results

BRAIN VOLUMES

Brain volumes are listed in table 2 . The volume of the following structures was significantly different in the Korsakoff group from that in the alcohol group and the control group: the hippocampus $(6 \%$ decrease compared with both the control and alcoholic group), the mamillary bodies (29\% decrease compared with both the control and alcoholic group), the third ventricle ( $72 \%$ increase compared with the control group and 38\% increase compared with the alcoholic group), and the thalamus (10\% decrease compared with both the control and alcoholic group). The parahippocampal gyrus was $5 \%$ smaller in both the Korsakoff group and the alcoholic group than in the control group $(p=0.07)$. The third ventricle tended to be $25 \%$ larger in the alcoholic group than in the control group $(\mathrm{p}=0.08)$.

CORRELATION BETWEEN BRAIN VOLUMES AND DELAYED RECALL IN THE KORSAKOFF GROUP The correlation coefficient between delayed recall and brain volume was significant for the third ventricle $(r=-0.54, p=0.05)$. None of the other correlations between delayed recall and brain structures reached significance. These correlations were -0.03 for the hippocampus $(p=0.93), 0.09$ for the mamillary bodies $(p=0.78)$, and 0.04 for the thalamus $(p=0.89)$.

\section{Discussion}

Volumetry on high resolution MRI showed that the volume of several brain structures that are involved in memory processing was decreased in subjects with Korsakoff's syndrome. The anterograde amnesia of the subjects with Korsakoff's syndrome correlated significantly with the volume of the third ventricle, suggesting that lesions in nuclei in the midline of the thalamus are responsible for the anterograde amnesia of these patients.

The involvement of the nuclei in the midline of the thalamus in the anterograde amnesia has also been reported in other studies of patients with Korsakoff syndrome. ${ }^{23}$ Of these midline nuclei, the mediodorsal nucleus has often been related to anterograde amnesia, ${ }^{28}$ but there are also studies that contradict the role of this structure in anterograde amnesia. ${ }^{4529}$ Other nuclei in the midline of the thalamus that may be involved in the anterograde amnesia of Korsakoff's syndrome are the parataenial nucleus, the paraventricular nucleus, the intermediodorsal nucleus, the reuniens nucleus, and the rhomboid nucleus. ${ }^{245}$ The severity of the anterograde amnesia may also depend on the number of nuclei that are affected. ${ }^{30}$
Although the volume of the mamillary bodies was reduced in most subjects in the Korsakoff group, it did not correlate with the severity of anterograde amnesia. This is by contrast with earlier studies that showed a positive relation between mamillary body size and memory. ${ }^{67}$ The discrepancy might be due to methodological shortcomings in these earlier studies because either the number of subjects was small $(n=4),{ }^{7}$ or the correlation was performed in a sample of alcoholics that included both subjects without cognitive impairment and subjects with severe cognitive impairment. ${ }^{6}$ Other studies have questioned the role of the mamillary bodies in anterograde amnesia. $^{231}$ Victor et al reported that some subjects with severe atrophy of the mamillary bodies were not amnesic. ${ }^{2}$ Shear et al showed that several patients with Korsakoff's syndrome and severe amnesia had no atrophy of the mamillary bodies. ${ }^{31}$ Thus atrophy of the mamillary bodies seems not to be sufficient or necessary to cause severe anterograde amnesia.

The medial temporal lobe seemed not to be involved in the anterograde amnesia. The volume of the hippocampus was modestly reduced in the subjects with Korsakoff's syndrome but it did not correlate with the score on the delayed recall task. A reduced volume of the hippocampus in Korsakoff's syndrome has been found in one neuropathological study. ${ }^{11}$ We found that the parahippocampal gyrus tended to be smaller in the alcoholics with or without Korsakoff's syndrome, which suggests that alcohol may have a neurotoxic effect on this structure.

We could not replicate the finding that atrophy of the mamillary body is common in alcoholics without amnesia. ${ }^{31} 32$ However, these studies used qualitative rating scales which are less accurate than volumetry. The alcoholics in our study did not have atrophy of the hippocampus, by contrast with other studies. ${ }^{22}$

One of the strong points of the study was that the brain structures were assessed with volumetry. Earlier neuroimaging studies used linear or qualitative measures, or did not follow anatomical boundaries. The exclusion criteria minimalised the potentially confounding effects of age and concomittant disorders that are often found in subjects with chronic alcoholism. A limitation of the study is that we did not assess all brain structures involved in memory processing such as the frontal lobe, the amygdala, and the cingulate gyrus. These structures may also be associated with the anterograde amnesia. Although our sample was large in comparison with that of other studies, 
the group size was still small and this has limited the power of the study. Correlational studies in general have the disadvantage that they show correlations and not causal relations. Studies using functional imaging techniques during neuropsychological testing may therefore further increase insight into the brain substrate of anterograde amnesia in alcoholic Korsakoff's syndrome.

In conclusion, this correlational study indicated that anterograde amnesia in alcoholic Korsakoff's syndrome is associated with atrophy of nuclei in the midline of the thalamus, but not with atrophy of the mamillary bodies, the hippocampus, or the parahippocampal gyrus.

We thank Dr T Wijdeveld for help in recruiting the subjects with alcoholism, and D Tisserand and S Kumar Rao for assisting in the measurement of the brain structures.

1 Kopelman M. The Korsakoff syndrome. Br f Psychiat 1995;166:154-73

2 Victor M, Adams R, Collins G. The Wernicke-Korsakoff syndrome and related neurologic disorders due to alcoholism and malnutrion. Philadelphia: FA Davis Company; 1989.

3 Shimamura A, Jernigan T, Squire L. Korsakoff's syndrome: radiological (CT) findings and neuropsychological correlates. F Neurosci 1988;8:4400-10.

4 Mair WG, Warrington EK, Weiskrantz L. Memory disorder in Korsakoff's psychosis: a neuropathological and neuropsychological investigation of two cases. Brain 1979;102: 749-83.

5 Mayes AR, Meudell PR, Mann D, et al. Location of lesions in Korsakoff's syndrome: neuropsychological and neuropathological data on two patients. Cortex 1988;24:36788 .

6 Estruch R, Bono G, Laine P, et al. Brain imaging in alcoholism. Eur $\mathcal{F}$ Neurology 1998;5:119-35.

7 ism. Eur F Neurology 1998;5:119-35. imaging of the hippocampal formation and mammillary imaging of the hippocampal formation and mammillary nuclei distinguish medial temporal lobe
amnesia. $\mathcal{F}$ Neurosci 1990;10:3106-17.

8 Joyce EM, Rio DE, Ruttimann UE, et al. Decreased cingulate and precuneate glucose utilization in alcoholic Korsakoff's syndrome. Psychiatry Res 1994;54:225-39.

9 Arendt T, Bigl V, Arendt A, et al. Loss of neurons in the nucleus basalis of Meynert in Alzheimer's disease, paralysi agitans and Korsakoff's disease. Acta Neuropathol 1983;61: 101-8.

10 Jernigan TL, Schafer $\mathrm{K}$, Butters $\mathrm{N}$, et al. Magnetic resonance imaging of alcoholic Korsakoff patients. Neuropsychopharmacology 1991;4:175-86.

11 Kril JJ, Halliday GM, Svoboda MD, et al. The cerebral cortex is damaged in chronic alcoholics. Neuroscience 1997;79: 983-98.
12 Deweer B, Lehéricy S, Pillon B, et al. Memory disorders in probable Alzheimer's disease: the role of hippocampal atrophy as shown with MRI. I Neurol Neurosurg Psychiatry 1995;58:590-7.

13 Jacobson R, Lishman W. Cortical and diencephalic lesions n Korsakoff's syndrome: a clinical and CT scan study. Psychol Med 1990;20:63-75.

14 American Psychiatric Association. Diagnostic and statistical manual of mental disorders. IVth ed. Washington, DC: APA; 1994.

15 Pfefferbaum A, Lim K, Zipursky R, et al. Brain gray and white volume loss accelarates with aging in chronic white volume loss accelarates with aging in chronic 1992;16:1078-89.

16 Krabbendam L, Visser P, Derix M, et al. Normal cognitive performance in patients with chronic alcoholism in contrast to patients with Korsakoff's syndrome. F Neuropsychiatry Clin Neurosci 1999 (in press).

17 Brand N, Jolles J. Learning and retrieval rates of words presented auditory and visually. F Gen Psychol 1985;112:20110.

18 Jolles J, Houx P, van Boxtel M, et al, eds. Maastricht aging study: determinants of cognitive aging. Maastricht: Neuropsych Publishers, 1995.

19 van Boxtel M, Buntinx F, Houx P, et al. The relation between morbidity and cognitive performance in a normal aging population. F Gerontol A Biol Sci Med Sci 1998;53: 146-54

20 Wechsler D. Wechsler adult intelligence scale. Manual. New York: Psychological Corporation, 1955.

21 Luteyn F, van der Ploeg F. Groninger intelligentie test. Lisse, The Netherlands: Swets en Zeitlinger, 1983.

22 Sullivan EV, Marsh L, Mathalon DH, et al. Anterior hippocampal volume deficits in nonamnesic, aging chronic alcoholics. Alcohol Clin Exp Res 1995;19:110-22.

23 Duvernoy H. The human hippocampus. An atlas of applied anatomy. München: JF Bergmann Verlag, 1988.

24 Duvernoy $\mathrm{H}$. The human brain: surface, three-dimensional sectional anatomy and MRI. Vienna: Springer-Verlag, 1991.

25 Jackson G, Duncan J. MRI neuroanatomy. A new angle on the brain. New York: Churchill Livingstone; 1996.

26 Lehéricy S, Baulac M, Chiras J, et al. Amygdalohippocampal MR volume measurements in the early stages of
Alzheimer's disease. AfNR Am $\mathcal{F}$ Neuroradiol 1994;15:92737.

27 Coffey C, Lucke J, Saxton J, et al. Sex differences in brain aging. Arch Neurol 1998;55:169-79.

28 Zola-Morgan S, Squire LR. Amnesia in monkeys after lesions of the mediodorsal nucleus of the thalamus. Ann Neurol 1985;17:558-64.

29 Markowitsch $H$. Thalamic mediodorsal nucleus and memory: a critical evaluation of studies in animals and man. Neurosci Biobehav Rev 1982;6:351-80.

30 Aggleton JP, Mishkin M. Memory impairments following restricted medial thalamic lesions in monkeys. Exp Brain Res 1983;52:199-209.

31 Shear PK, Sullivan EV, Lane B, et al. Mammillary body and cerebellar shrinkage in chronic alcoholics with and without amnesia. Alcohol Clin Exp Res 1996;20:1489-95.

32 Blansjaar B, Vielvoye G, van Dijk J, et al. Similar brain lesions in alcoholics and Korsakoff patients: MRI, psychometric and clinical findings. Clin Neurol Neurosurg 1992;94: 197-203. 